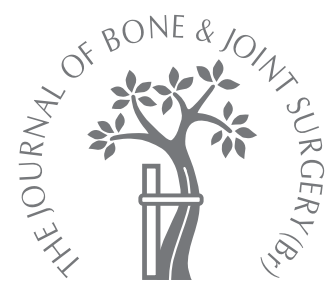

\title{
The use of patient-reported outcome measures and patient satisfaction ratings to assess outcome in hemiarthroplasty of the shoulder
}
J. L. Rees,
J. Dawson,
G. C. R. Hand,
C. Cooper,
A. Judge,
A. J. Price,
D. J. Beard,
A. J. Carr

From the University of

Oxford, Oxford,

United Kingdom

J. L. Rees, MBBS, FRCS

(Eng), MD, FRCS (Orth),

University Lecturer in

Orthopaedics, Honorary

Consultant in Shoulder and

Elbow Surgery

C. Cooper, RGN, Clinical

Research Nurse

A. Judge, BSc, MSc, PhD,

Senior Statistician

A. J. Price, BSc, MSc, PhD,

Clinical Reader in

Musculoskeletal Science

D. J. Beard, DPhil, University

Lecturer and RCUK Fellow

A. J. Carr, MA, ChM, FRCS,

FMedSci, Professor of

Orthopaedic Surgery

Nuffield Department of

Orthopaedics, Rheumatology

and Musculoskeletal Sciences

NIHR Biomedical Research

Unit, University of Oxford,

Oxford OX3 7LD, UK.

J. Dawson, DPhil, Senior

Research Scientist

Department of Public Health

University of Oxford, Old Road

Campus, Old Road, Oxford OX3 7LF, UK.

a G. C. R. Hand, MB, BS, FRCS

(Eng), FRCS (Orth), Consultant

Orthopaedic Surgeon

Southampton University

Hospital, Tremona Road,

Southhampton SO16 6YD, UK.

Correspondence should be sent

to Mr J. L. Rees; e-mail:

jonathan.rees@ndorms.ox.ac.uk

(C)2010 British Editorial Society of Bone and Joint Surgery doi:10.1302/0301-620X.92B8. $22860 \$ 2.00$

$J$ Bone Joint Surg $[B r]$ 2010;92-B:1107-11. Received 2 June 2009; Accepted after revision 13 April 2010

We have compared the outcome of hemiarthroplasty of the shoulder in three distinct diagnostic groups, using survival analysis as used by the United Kingdom national joint registers, patient-reported outcome measures (PROMs) as recommended by Darzi in the 2008 NHS review, and transition and satisfaction questions.

A total of 72 hemiarthroplasties, 19 for primary osteoarthritis (OA) with an intact rotator cuff, 22 for $O A$ with a torn rotator cuff, and 31 for rheumatoid arthritis (RA), were followed up for between three and eight years. All the patients survived, with no revisions or dislocations and no significant radiological evidence of loosening. The mean new Oxford shoulder score (minimum/worst 0 , maximum/best 48 ) improved significantly for all groups $(p<0.001$ ), in the OA group with an intact rotator cuff from 21.4 to 38.8 (effect size 2.9), in the OA group with a torn rotator cuff from 13.3 to 27.2 (effect size 2.1) and in the RA group from 13.7 to 28.0 (effect size 3.1). By this assessment, and for the survival analysis, there was no significant difference between the groups. However, when ratings using the patient satisfaction questions were analysed, eight $(29.6 \%)$ of the RA group were 'disappointed', compared with one $(9.1 \%)$ of the OA group with cuff intact and one $(7.7 \%)$ of the OA group with cuff torn. All patients in the OA group with cuff torn indicated that they would undergo the operation again, compared to ten $(90.9 \%)$ in the OA group with cuff intact and $20(76.9 \%)$ in the RA group.

The use of revision rates alone does not fully represent outcome after hemiarthroplasty of the shoulder. Data from PROMs provides more information about change in pain and the ability to undertake activities and perform tasks. The additional use of satisfaction ratings shows that both the rates of revision surgery and PROMs need careful interpretation in the context of patient expectations.

Degenerative changes in joints represent a significant health burden worldwide, and their prevalence is expected to rise. ${ }^{1-4}$ This will increase the demand for arthroplasty. ${ }^{5-8}$ Most developed countries have national joint registers monitoring the outcome of joint replacements. The results, generally presented as revision rates or formal survival analyses, represent outcomes and performance based on the use of revision as the endpoint. Although pain may be an indication for revision, Murray and Frost, ${ }^{9}$ in 1998 showed that when pain, instead of revision, was used as an endpoint in survival analysis, the success of total knee replacement dropped from $97.5 \%$ to $72 \%$. This demonstrates that lack of revision alone does not necessarily mean the continued 'success' of an operation or an implant.

Besides using revision as an assessment of outcome, clinician-based scores have remained the most popular adjunct to survival analysis.
However, these are prone to observer bias. ${ }^{10}$ Orthopaedic surgery has started to follow the non-surgical specialties by developing and using outcome assessments that are completed by patients. The use of these validated patientreported outcome measures (PROMs) has, however, remained limited, despite the availability of a number of suitable scores with satisfactory measurement properties. ${ }^{11-14}$ PROMs are intended to assess outcome from the patient's perspective and can vary in their specificity. Some are generic (such as the ShortForm $\left.36(\mathrm{SF}-36)^{15}\right)$, whereas others are disease-specific, such as the Arthritis Impact Measurement Scales, ${ }^{16}$ and limb-specific, such as the Disabilities of the Arm, Shoulder and Hand (DASH). ${ }^{12}$ The Oxford scores (hip, knee, shoulder and elbow) were devised with the help of patients who were about to undergo surgery. They were designed as joint-specific PROMs and validated in the context of surgery. They 
represent the perspective of the patient rather than of the clinician. Their importance in elective orthopaedics has recently been highlighted by a review from the United Kingdom Department of Health. ${ }^{17}$ The Oxford scores have been used since April 2009 to assess the outcome of hip and knee replacements in the United Kingdom, ${ }^{18-20}$ and assessment of other joint replacements, such as the shoulder, using PROMs will shortly be introduced. PROMs have also been chosen for this role because they are postal questionnaire scores with high return rates and are therefore more costeffective than calling patients back for assessment.

However, any methods used to evaluate treatment must have been developed appropriately and assessed for acceptability by the patients, reliability, validity and responsiveness. The evidence for these properties must have been collected in a similar context and on similar types of patient. Most PROMs, including the Oxford scores, have been designed for use in trials where a specific hypothesis is being tested with predetermined inclusion and exclusion criteria. The widespread use of PROMs, as proposed by Darzi in the NHS Next Stage Review, ${ }^{17}$ may offer advantages over either the absence of any outcome data or of limited data based on rates of revision surgery alone. However, this widespread 'audit' will lead to comparisions being made between surgeons, centres and types of procedure, which could be misleading. For example, for some patients with certain conditions a treatment may be provided with both patient and clinician being aware that the outcome or expectations are limited. However, as long as these are achieved, the patient will probably be satisfied, irrespective of the final outcome score. If these scores are to be used in large-scale audits of practice where the case mix is heterogeneous and individual expectations of patients may vary, adding or anchoring established PROMs with transition and satisfaction questions is likely to be necessary in order to interpret and fully understand the outcome after joint replacement.

The aim of this observational study was to identify any variation observed when different methods of assessment were used to compare the outcome of hemiarthroplasty of the shoulder in three distinct diagnosic groups. Two established methods of assessment were used, namely survival analysis as used by the national joint registers and a PROM as recommended by Darzi, ${ }^{17}$ together with patient transition and satisfaction questions.

\section{Patients and Materials}

Between 1998 and 2003, 103 primary shoulder hemiarthroplasties were performed by one surgeon in patients with primary OA and RA of the glenohumeral joint, using the Oxford Shoulder Replacement (Corin, Cirencester, United Kingdom). Pain was the overriding indication for surgery. None of the patients had less than $80^{\circ}$ of active elevation and none had significant superior migration of the humeral head or a history of previous surgery. The integrity of the rotator cuff was assessed under direct vision at operation as either intact or torn. Only full-thickness tear of $\geq 5 \mathrm{~cm}$ was defined as a cuff tear. None were repaired. All patients had the same standard programme of postoperative rehabilitation.

The primary outcome measure was the new validated Oxford Shoulder Score (OSS), ${ }^{21}$ which assesses the symptoms and function experienced during the preceding four weeks. It is appropriate for assessing all shoulder surgery except where conducted for instability. ${ }^{22,23}$ It contains 12 items, each with five response options, scored from 0 to 4 , with 0 representing the worst response. Scores from individual items are added to produce a single figure ranging from 0 to 48, where a score of 0 represents the worst possible outcome to the patient. The original OSS scored each item from 1 to 5 , from least to most difficulty or severity. These were added to produce a single score with a range from 12 (fewest difficulties) to 60 (most difficulties). The recommended method of scoring was recently revised to 0 to 48 to make the system more intuitive and in line with similar changes made to the Oxford Hip and Knee Scores. The old OSS has been shown to be internally consistent, reliable, valid and sensitive to clinical change. ${ }^{21}$ The Oxford shoulder scores were collected prospectively, immediately prior to surgery, and after a minimum follow-up of three years ( 3 to 8$)$. The scores were analysed by a senior statistician (AJ).

Patients also completed one transition- and two satisfaction-related questions. The transition question was: How are the problems related to your shoulder NOW, compared with before surgery? (response options: no problems at all, much better, slightly better, no change, slightly worse, much worse). The satisfaction questions were: Overall, how pleased have you been with the result of your surgery so far? (response options: very pleased, fairly pleased, not very pleased, very disappointed), and, if you could go back in time, would you still choose to have the shoulder operation? (response options: yes, no, not sure).

Radiographs were taken pre-operatively, within two days of surgery and again at a minimum of three years after operation. Standard anteroposterior (AP) and axillary lateral views were assessed for glenoid erosion and stem loosening. The proximal humerus was divided into eight zones for assessment of lucency, as previously described. ${ }^{24,25}$ Loosening was defined as $>2 \mathrm{~mm}$ of lucency around the stem in any zone, and gleniod erosion defined as a horizontal loss of glenoid bone stock of more $>2 \mathrm{~mm}$. Radiographs were analysed by three experienced observers (AJC, GCRH and an independent observer), who had to agree on the diagnosis.

Statistical analysis. The pre-operative, post-operative and difference in OSS scores were assessed for normality using the Shapiro-Wilk test. There were no major departures from normality. The mean and SD were therefore used as summary statistics for each group. Paired $t$-tests were used to compare the pre- and post-operative OSS. Unpaired $t$-tests were used to assess whether the difference in OSS varied between the three groups; OA intact, OA torn, RA. 


\begin{tabular}{|c|c|c|c|c|}
\hline & All & OA intact & OA torn & RA \\
\hline Age (mean, SD) & $69.9(11.1)$ & $72.2(6.9)$ & $77.0(7.2)$ & $63.5(11.9)$ \\
\hline \multicolumn{5}{|l|}{ Gender, n (\%) } \\
\hline Female & $60(83.3)$ & $15(79.0)$ & $20(90.9)$ & $25(80.7)$ \\
\hline Male & $12(16.7)$ & $4(21.1)$ & $2(9.1)$ & $6(19.4)$ \\
\hline Total & 72 & 19 & 22 & 31 \\
\hline
\end{tabular}

Table II. Outcome of uncemented hemiarthroplasty: Oxford Shoulder Score (OSS)

\begin{tabular}{|c|c|c|c|c|}
\hline & OSS pre-operative & OSS post-operative & $\begin{array}{l}\text { OSS follow-up period } \\
\text { (months) }\end{array}$ & $\begin{array}{l}\text { Radiographic follow- } \\
\text { up period (months) }\end{array}$ \\
\hline \multicolumn{5}{|l|}{ Total $(n=72)$} \\
\hline Mean (range) & 15.6 (4 to 33 ) & $30.6(10$ to 48$)$ & 53.5 (36 to 96$)$ & $52.0(20$ to 86$)$ \\
\hline \multicolumn{5}{|c|}{$\mathrm{OA}^{*}$ cuff intact $(n=19)$} \\
\hline Mean (range) & 21.4 (11 to 33 ) & 38.8 (17 to 48 ) & 54.6 (36 to 86$)$ & 54.4 (20 to 86$)$ \\
\hline \multicolumn{5}{|c|}{ OA cuff tear $(n=22)$} \\
\hline Mean (range) & 13.3 (4 to 25$)$ & 27.2 (10 to 46$)$ & 53.8 (36 to 72 ) & 51.8 (26 to 73$)$ \\
\hline \multicolumn{5}{|l|}{$\mathrm{RA}^{\dagger}(\mathrm{n}=31)$} \\
\hline Mean (range) & 13.7 (4 to 24 ) & 28.0 (12 to 48$)$ & 52.5 (36 to 96$)$ & 50.6 (30 to 78$)$ \\
\hline
\end{tabular}

* OA, osteoarthritis

$\uparrow \mathrm{RA}$, rheumatoid arthritis

Effect sizes were calculated for the OSS. The effect size is a method of calculating the magnitude of change, or treatment effect, that has occurred. ${ }^{26}$ In this study it was measured as the difference between the mean pre- and post-operative scores, divided by the SD of pre-operative scores. An effect size of 1.0 is equivalent to a change of one SD in the sample, which is considered to be a very large change, and an effect size of 0.8 is considered large, 0.5 moderate and 0.2 small. Fisher's exact tests were used to compare transition and satisfaction results between diagnosis groups.

\section{Results}

A total of 103 uncemented hemiarthroplasties of the shoulder were performed in 96 patients; 24 patients had died from unrelated causes. The last radiograph prior to their death revealed no radiological evidence of failure or erosion. Seven further patients were not contactable, leaving 72 shoulders in 65 patients. Of these, 12 were in men and 60 in women. Their mean age was 69.9 years (27 to 88). There were 41 replacements in OA patients, 19 with an intact rotator cuff and 22 with a torn cuff. The remaining 31 replacements were for RA and these patients had thin, atrophic or torn tendons in the rotator cuff consistent with long-term chronic joint disease (see Table I for subgroup demographics). Standard AP and axillary radiographs were available for all patients at a minimum of three years postoperatively. In one shoulder there was evidence of minor lucency $<2 \mathrm{~mm}$ in the zone of the greater tuberosity) but no patients required revision surgery or suffered a dislocation, infection or nerve damage.

Oxford shoulder scores. Prospective OSS data were available for all 72 cases for a minimum of three years ( 3 to 8 ) (Table II). The mean OSS improved from 15.6 to 30.6 overall (effect size 2.3). In the OA group it improved from 21.4 to 38.8 in the cuff-intact group (effect size 2.9) and from 13.3 to 27.2 in the cuff-torn group (effect size 2.1). In patients with RA the mean OSS improved from 13.7 to 28.0 (effect size 3.1). All these changes in OSS within the group were highly significant $(\mathrm{p}<0.001)$. Subsequent pairwise comparisons using unpaired $t$-tests revealed no statistically significant improvement in the OSS of one group over another (Table III).

Transition and satisfaction questions. The transition and satisfaction questionnaires were completed by 51 patients $(78 \%)$. The transition response revealed that $11(21.6 \%)$ were worse or the same after surgery, while $3(5.9 \%)$ were slightly better, and $37(72.6 \%)$ much better. The satisfaction responses indicated that ten $(19.6 \%)$ were very disappointed or not pleased, and $41(80.4 \%)$ were fairly or very pleased. A total of 44 patients $(86.3 \%$ ) said that, given the time again, they would have had the operation whereas seven $(13.7 \%)$ would have declined or were unsure.

The responses varied between subgroups. For the transition responses in the OA cuff-intact group, one $(9.1 \%)$ was 
Table III. Change in mean Oxford Shoulder Score (OSS) by diagnostic group (no significant differences between groups)

\begin{tabular}{lllll}
\hline Group $^{*}$ & Number & Change in mean OSS & Pre-operative OSS (SD) & Effect size \\
\hline OA rotator cuff intact & 19 & 17.4 & 5.9 & 2.9 \\
OA rotator cuff tear & 22 & 13.9 & 6.5 & 2.1 \\
RA & 31 & 14.3 & 4.6 & 3.1 \\
\hline
\end{tabular}

* OA, osteoarthritis; RA, rheumatoid arthritis

worse or the same, whereas two $(18.2 \%)$ felt slightly better and eight $(72.7 \%)$ felt much better. In the OA cuff-torn group, one $(7.7 \%)$ was worse or the same, one $(7.7 \%)$ was slightly better and $11(84.6 \%)$ were much better. For the RA group, nine $(33.3 \%)$ were worse or the same and $18(66.7 \%)$ were much better. For the satisfaction responses in the OA cuff-intact group, one $(9.1 \%)$ was not very pleased, whereas three $(27.3 \%)$ were fairly pleased and seven $(63.6 \%)$ were very pleased. In the OA cuff-torn group, one $(7.7 \%)$ was not very pleased, two $(15.4 \%)$ were fairly pleased and ten $(76.9 \%)$ were very pleased. In the RA group two $(7.4 \%)$ were very disappointed, six $(22.2 \%)$ were not very pleased, four $(14.8 \%)$ fairly pleased and 15 $(55.6 \%)$ very pleased. All patients in the OA cuff-torn group would have surgery again, in the OA cuff-intact group ten $(90.9 \%)$ would have surgery again, but in the RA group only $20(76.9 \%)$ would have surgery again.

A Fisher's exact test comparing the transition and satisfaction responses between groups revealed the RA group to be more disappointed $(\mathrm{p}=0.056)$ and less likely to have surgery again than the OA cuff-intact and cuff-torn groups $(\mathrm{p}=0.055)$.

\section{Discussion}

The assessment of joint replacements in the United Kingdom is now conducted by survival analysis and PROMs using Oxford scores. Applying the appropriate outcome measures is important as it allows us to assess the efficacy of an intervention, to select effective treatments and may assist with monitoring improvement or deterioration over time for each individual patient. However, most outcome measures are not designed to assess individual patients and need to be sufficiently precise and reproducible to allow for this application. ${ }^{27}$ There is now growing enthusiasm for PROMs. They have clear advantages over clinician-based scores, which are prone to bias, invariably describe a more successful outcome, have higher rates of loss to follow-up, and are more costly. ${ }^{28,29}$ Joint replacement improves the quality of life and PROMs more accurately reflect this than do clinician-rated scores, which place an emphasis on range of movement and power, or more generic patient-reported outcome scores which are more easily affected by comorbidity.

In an attempt to improve the assessment of outcome, we examined of three subgroups of patients undergoing uncemented hemiarthroplasty of the shoulder using three different methods of assessment, two of which are currently used for national data. There have been no prospective comparative studies of outcome in these subgroups, and no studies that have compared the outcome if different methods of assessment are used.

Using survival analysis alone, with revision as an endpoint, to analyse outcome in smaller studies with shorter periods of follow-up, such as ours, can be misleading, as no implants failed and the results therefore appear excellent. In larger national joint registers, survival analysis using revision rates is useful to identify specific implant-related failures, but overall, in whatever setting this type of analysis is used, it fails to give information about patient satisfaction.

In our study, when the outcome from the patient's perspective is examined using PROMs, results were less favourable than suggested by the $0 \%$ revision rate. All patients had poor scores pre-operatively, and in all three groups hemiarthroplasty resulted in a significant improvement in OSS scores. However, an 'improved' score is not necessarily synonymous with a 'good' final score, and where patients start from a low base there is plenty of scope for improvement that may nonetheless fall short of a 'good outcome'. When further patient perspective was sought using transition and satisfaction responses, they added surprising descriptive value to the assessment of outcome, highlighted by the finding that some patients were not pleased with their outcome, which was not necessarily reflected in a low PROM score.

The analysis of the change in the OSS revealed that no one diagnostic group had improved significantly more than another. There were nevertheless differences between the groups in the responses to the transition and satisfaction questions. The RA group were less likely to feel better following surgery, to be more disappointed with the outcome and less likely to want to undergo the procedure again. It is possible that this was a feature of systemic versus local pathology, and that although the joint-specific pain improved, limb and bodily function did not. When the OA cuff-torn group was compared to the OA cuff-intact group they did less well, both by final score $(27.2 v s 38.8)$ and by mean change in score $(14.1$ vs 16.4$)$, but $76.9 \%$ were 'very pleased' compared with $63.6 \%$ in the OA cuff-intact group. It may be that in this slightly older cohort, expectations were different. It is plausible that during the consent process it was agreed by patient and surgeon that the goal was improvement in pain with no significant expectation of gain in function. Whatever the reasons for these observed 
and surprising differences, the transition and satisfaction responses have added information that was not detected by a validated PROM.

It is evident that using revision as the sole endpoint may not reflect the patients' perceptions of success. In this cohort no revisions were performed, and yet $19.6 \%$ of patients were either very disappointed or not very pleased. The descriptive information that simple patient satisfaction questions provided has added value. The return rate for these questions, however, was lower than the $100 \%$ return of the OSS, which is of concern, but we feel this can be rectified by simple changes to the formatting. These patient satisfaction questions were used in the development of all the Oxford scores and we now use them in addition to the OSS for all groups of patients, as they provide additional useful information regarding the efficacy of treatment. In the future we plan to compare patient satisfaction to the change in the PROM score to determine whether threshold value and risk adjustment factors can be developed to allow useful comparisons of patient subgroups.

The widespread use of PROMs in regional and national audits of outcome of orthopaedic operations is under way in the United Kingdom and is planned in other countries. This is a considerable undertaking with substantial associated costs. An understanding of the effectiveness of orthopaedic operations is clearly a laudable aim. However, great care needs to be taken in planning these studies so that meaningful interpretations can be made which are useful to patients, clinicians and funders of health care. What data should be collected, and how frequently? What is an acceptable minimum follow-up? How should procedures, surgeons and centres be compared? The routine use of PROMs in health care settings will therefore need to be established carefully. ${ }^{30}$ There will be groups of patients and types of procedure where these data are liable to misinterpretation if the expectations of individual patients are not understood. Arguably, joint replacement surgery represents one of the most straightforward of operations, but this study shows that rates of revision and the PROMs scores alone do not adequately reflect the expectations and level of satisfaction of patients with different pathologies undergoing the same procedure.

One or more of the authors have received or will receive benefits for persona or professional use from a commercial party related directly or indirectly to the subject of this article.

\section{References}

1. Reginster JY. The prevalence and burden of arthritis. Rheumatology (Oxford) 2002;41(Supp|1):3-6.

2. No authors listed. The Centres for Disease Control and Prevention. Prevalence of self-reported arthritis or chronic joint symptoms among adults - United States 2001 JAMA 2002;288:3103-4.
3. Lawrence RC, Felson DT, Helmick CG, et al. Estimates of the prevalence of arthritis and other rheumatic conditions in the United States: Part II. Arthritis Rheum 2008;58:2635.

4. Helmick CG, Felson DT, Lawrence RC, et al. Estimates of the prevalence of arthritis and other rheumatic conditions in the United States: Part I. Arthritis Rheum 2008:58:1525.

5. Kurtz S, Mowat F, Ong K, et al. Prevalence of primary and revision total hip and knee arthroplasty in the United States from 1990 through 2002. J Bone Joint Surg [Am] 2005:87-A:1487-97.

6. Adams JE, Sperling JW, Hoskin TL, Melton LJ 3rd, Cofield RH. Shoulder arthroplasty in Olmsted County, Minnesota, 1976-2000: a population-based study. J Shoulder Elbow Surg 2006;15:50-5.

7. Fear J, Hillman M, Chamberlain MA, Tennant A. Prevalence of hip problems in a population aged 55 years and over: access to specialist care amd future demand for hip arthroplasty. Br J Rheumatol 1997;36:74-6.

8. No authors listed. Joint replacement: demand and future projections. http:// www.cdc.gov/arthritis/data_statistics/state_data.htm (date last accessed 20 March 2010).

9. Murray DW, Frost SJ. Pain in the assessment of total knee replacement. J Bone Joint Surg [Br] 1998;80-B:426-31.

10. Pynsent P, Fairbank J, Carr A. Outcome measures in orthopaedics . Oxford: Butterworth-Heinemann, 1993.

11. Roach KE, Budiman-Mak E, Songsiridej N, Lertratanakul Y. Development of a shoulder pain and disability index. Arthritis Care Res 1991;4:143-9.

12. Hudak PL, Amadio PC, Bombardier C. Development of an upper extremity outcome measure: the DASH (disabilities of the arm, shoulder and hand) [corrected]. Am J Ind Med 1996;29:602-8.

13. Kirkley A, Griffin S, Dainty K. Scoring systems for the functional assessment of the shoulder. Arthroscopy 2003;19:1109-20.

14. Cloke DJ, Lynn SE, Watson H, et al. A comparison of functional, patient-based scores in subacromial impingement. J Shoulder Elbow Surg 2005;14:380-4.

15. Ware JE Jr, Sherbourne CD. The MOS 36-item short-form health survey (SF-36). I: conceptual framework and item selection. Med Care 1992;30:473-83.

16. Meenan R GP, Mason J. Measuring health status in rheumatoid arthritis: the Arthritis Impact Measurement Scales. Arthritis Rheum 1980;23:146-52.

17. Darzi A. NHS Next Stage Review Interim Report: Survey October 2007. London: Department of Health, 2007

18. Dawson J, Fitzpatrick R, Carr A, Murray D. Questionnaire on the perceptions of patients about total hip replacement. J Bone Joint Surg [Br] 1996;78-B:185-90.

19. Dawson J, Fitzpatrick R, Murray D, Carr A. Questionnaire on the perceptions of patients about total knee replacement. J Bone Joint Surg [Br] 1998;80-B:63-9.

20. Murray DW, Fitzpatrick $\mathbf{R}$, Rogers $\mathbf{K}$, et al. The use of the Oxford hip and knee scores. J Bone Joint Surg [Br] 2007;89-B:1010-14.

21. Dawson J, Fitzpatrick R, Carr A. Questionnaire on the perceptions of patients about shoulder surgery. J Bone Joint Surg [Br] 1996;78-B:593-600

22. Dawson J, Fitzpatrick R, Carr A. The assessment of shoulder instability: the development and validation of a questionnaire. J Bone Joint Surg [Br] 1999;81-B:420-6.

23. Moser JS, Barker KL, Doll HA, Carr AJ. Comparison of two patient-based outcome measures for shoulder instability after nonoperative treatment. J Shoulder Elbow Surgery 2008; 17:886-92

24. Mileti J, Boardman ND 3rd, Sperling JW, et al. Radiographic analysis of polyethylene glenoid components using modern cementing techniques. J Shoulder Elbow Surg 2004:13:492-8.

25. Sanchez-Sotelo J, O'Driscoll SW, Torchia ME, Cofield RH, Rowland CM. Radiographic assessment of cemented humeral components in shoulder arthroplasty. $J$ Shoulder Elbow Surg 2001;10:526-31.

26. Kazis LE, Anderson JJ, Meenan RF. Effect sizes for interpreting changes in health status. Med Care 1989;27(Suppl):178-89

27. McHorney CA, Tarlov AR. Individual-patient monitoring in clinical practice: are available health status surveys adequate? Qual Life Res 1995;4:293-307.

28. Fitzpatrick R, Davey C, Buxton MJ, Jones DR. Evaluating patient-based outcome measures for use in clinical trials. Health Technol Assess 1998;2:1-74.

29. Timmins N. NHS goes to the PROMS. BMJ 2008;336:1464-5.

30. Dawson J, Doll H, Fitzpatrick R, Jenkinson C, Carr AJ. The routine use of patient reported outcome measures in healthcare settings. BMJ 2010;340:c186.doi 10.1136. 